\title{
Los refranes de Celestina interpretados por su primer comentarista
}

\author{
Hugo O. Bizzarri
}

Université de Fribourg

\begin{abstract}
A nadie escapa que en las últimas décadas los estudios celestinescos han sufrido dos cambios radicales. Primeramente, el descubrimiento de Charles Faulhaber del manuscrito de Palacio conservando copia manuscrita del "primitivo aucto", que ha renovado las disputas sobre la génesis de la obra y problemas de filiación de las primitivas impresiones. ${ }^{1}$ En segundo lugar, la publicación del manuscrito de la Biblioteca Nacional de Madrid 17631, comúnmente llamado Celestina comentada, conocido desde antiguo, utilizado por estudiosos y comentaristas de la obra, pero que hasta el momento estaba sólo a disposición de quienes tuvieran tiempo de consultarlo en sus estancias científicas madrileñas. Su publicación en el año 2002, fruto de un equipo de trabajo, permite hoy tener acceso fácil y directo a esta forma de recepción de la obra de Rojas. ${ }^{2}$

Aspectos claves de este comentario, tales como la fecha de su elaboración, autoría y profesión del autor están actualmente en disputa. De todo ello, parece haber acuerdo general en los críticos de que este anónimo glosador era un jurista, posiblemente influido por las ideas del mos gallicus, corriente originaria de Francia, que estaba de moda en España en la primera mitad del siglo XVI. ${ }^{3}$ Más ambigua es la fecha de elaboración
\end{abstract}

1.- Charles B. Faulhaber, "Celestina de Palacio: Madrid, Biblioteca de Palacio, Ms. 1520», Celestinesca 14 n² (1990), pp. 3-39. Volvió sobre el tema en «Celestina de Palacio: Roja's Holograph Manuscript?» Celestinesca, 15 n 1 (1991), pp. 3-52.

2.- Louise Fothergill-Payne, Enrique Fernández Rivera y Peter Fothergill-Payne (eds.), Celestina comentada, Salamanca, Ediciones Universidad de Salamanca, 2002. Las citas estarán hechas de esta edición.

3.- El primero en señalar la profesión de este comentarista fue Peter Russell en dos trabajos "El primer comentario crítico de La Celestina: cómo un legista del siglo XVI interpretaba la Tragicomedia", en Temas de La Celestina y otros estudios. Del Cid al Quijote, Barcelona, Ariel, 1978, pp. 293-321 y «La Celestina y los estudios jurídicos de Fernando de Rojas», ibidem, pp. 325-340. 
de este comentario para lo cual los estudiosos se han valido de los libros que el mismo glosador cita a lo largo de su trabajo. Russell observó que en una glosa al acto IV menciona a Juan de Dios, fundador del hospital de pobres de Granada: "Los términos de la nota sugieren que fue escrito algunos años después de 1550; quizá antes de la bula papal de 1571, y casi con seguridad antes de la publicación de la biografía de $1585 » .^{4}$ Louise Fothergill-Payne llega casi a la misma conclusión al observar que la biblioteca de este intelectual estaba constituida por obras publicadas entre 1520 y 1570 . El autor podría haber iniciado su comentario entre 1550 o comienzos de 1560, alcanzando el estado en que nos ha quedado, tal vez por muerte de su autor, en $1570 .^{5}$

Otra observación importante de Russell concierne a la patria chica del comentarista. Para el crítico británico, la glosa del acto Iv a la que acabamos de hacer referencia, evidencia que debió de escribirse en Granada. ${ }^{6}$ Sin embargo, Fernández Rivera acota acertadamente que es curioso que el comentarista pase por alto las palabras que dice Sempronio en el mismo acto, "ganada es Granada", sin dedicarles una glosa, totalmente esperable si estuviera escribiendo en dicha ciudad. ${ }^{7}$

Quisiera aclarar desde ahora que mis apreciaciones sobre esta copia no están destinadas a solucionar ninguno de estos puntos. Más bien me interesa mirar a este comentario desde otra óptica. Louise Fothergill-Payne señaló con certeza que este comentarista anónimo prefiere glosar pasajes sentenciosos, ${ }^{8}$ a lo cual habría que añadir que gran parte de ellos son refranes. Los críticos hasta ahora han acentuado la relación de estos comentarios con el derecho tanto canónico como seglar, especialmente con la intención de describir el perfil del comentarista. Ahora yo quisiera prestar atención a otro elemento que se desprende de las glosas: la percepción que este comentarista tenía de la lengua castellana.

El primer punto que quisiera destacar es que para los años 60 ó 70 del siglo XVI no era extraña esta forma de comentar los refranes que presenta el anónimo comentarista de Celestina ni que este tipo de trabajo fuera realizado por un entendido en Derecho. La más antigua muestra hispánica

Han hecho nuevas precisiones Ivy Corfis, «La Celestina comentada y el código jurídico de Fernando de Rojas", en A. Deyermond y I. Macpherson (eds.), The Age of the Catholic Monarchs 1474-1516: Literary Studies in Memory of Keith Whinnom. Número especial del Bulletin of Hispanic Studies, Liverpool, Liverpool UP, 1989, pp. 19-24 y E. Fernández Rivera, "La autoría y el género de la Celestina comentada» RFE, $86 \mathrm{n}^{\circ} 2$ (2006), pp. 259-276.

4.- Russell, "El primer comentario crítico...», p. 301.

5.- Louise Fothergill-Payne, "Introducción», en Louise Fothergill-Payne et alii, Celestina comentada, pp. XV y XVIII-XXI.

6.- Russell, «El primer comentario crítico...», pp. 300-301.

7.- Fernández Rivera, «La autoría...», p 263 nota 11.

8.- Louise Fothergill-Payne, "Introducción», en Louise Fothergill-Payne et alii, Celestina comentada, pág. XVII. 
de esta forma de glosar la hallamos en el Seniloquium, pequeña colección de refranes glosados en latín que fue escrita entre 1478 y $1480 .{ }^{9}$ Su autor se vale para comentar los refranes de gran cantidad de auctoritates, pero también de costumbres, relatos populares y hasta de refranes. Entre los relatos populares que recrea no podemos dejar de pasar por alto el del refrán 436, "Quien echara el çençerro al gato", relato que conocemos por su inserción en el Libro de los gatos (ej. n 55 ) y el n 328 , «Piensa el ladrón que todos son de su condición", que trae en su comentario el difundido cuento de la «Disputa entre griegos y romanos» que se halla en el Libro de buen amor (cc. 44-70). El anónimo comentarista y compilador del Seniloquium quiere amonestar al bajo clero y para ello destaca la moralidad de estas paremias, muchas veces interpretadas de forma harto personal. Para ello hace gala de una amplia erudición en que destacan sus conocimientos tanto del Derecho seglar como del canónico. Doy como ejemplo el comentario al refrán «A yra de Dios, non ay cosa fuerte» $\left(n^{\circ} 24\right)$ :

Porque un reo no encuentra ayuda ante una legación, en la que se presenta un testigo de los propios hechos; por ello es ejecutado por el juez. Nadie puede ocultarse en sitio alguno a los ojos del juez que lo ve todo. En vano huye uno y se esconde de la mirada de Dios, pues se ha dicho: Si asciendo al cielo, tú estás allí. Sobre este versículo comentó mejor Anselmo estos versos: :para qué huyes, alma? Por muchas horas que subas, siempre estarás bajo la mirada de Dios. Y no piensen los hombres que el poder de las riquezas prevalecerá en su presencia. En los comentaristas encontramos igual explicación. ${ }^{10}$

En este escueto comentario se han esgrimido nociones de derecho seglar, citas de versículos bíblicos con los cuales se corrobora la sentencia, es decir, el sentido profundo del refrán, y la tradición patrística. Todo esto para extraer una enseñanza cristiana del dicho popular. No voy a detenerme más en este texto, pero constantemente la tradición escolástica sirve a este anónimo comentarista para corroborar la moralidad de los refranes. La sabiduría popular no hace más que transmitir de otra forma lo que habían dicho los doctores de la iglesia y hasta el mismo texto bíblico.

El compilador y glosador del Seniloquium pertenece al ámbito escolar y hunde sus raíces en esa tradición, en la cual los refranes eran utilizados en las clases de gramática. Colecciones de este tipo con comentarios no

9.- Es la hipótesis que manejan actualmente Fernando Cantalapiedra y Juan Moreno (eds.), Diego García de Castro. Seniloquium, Valencia, Universidad de Valencia, 2006, p. 26. En otra aportación, Fernando Cantalapiedra Erostarbe («La Celestina y Seniloquium», Celestinesca, 29 (2005), pp. 9-44) ha reseñado unos 150 pasajes comunes entre Seniloquium y Celestina sin que esto implique influencia, préstamos o dependencias.

10.- Me valgo de la traducción de Cantalapiedra y Moreno (eds.), Seniloquium, p. 66. 
escasean en la Edad Media ${ }^{11}$ y de ella se va a nutrir Erasmo para elaborar sus Adagia y transformar a los refranes en vehículos de su reforma.

Pero entre el Seniloquium y el comentario de Celestina ha habido un cambio radical. En el interín se ha producido un proceso de jerarquización de la lengua castellana. Sabemos que este proceso tiene su raíz en las disputas que se realizaban en Italia entre lengua latina y vulgar. Aunque en España no se hayan desarrollado con la misma magnitud, no faltaron en la Península Ibérica una larga serie de reflexiones en torno a la lengua vulgar, en las cuales los refranes no han quedado al margen. ${ }^{12}$ Mal Lara dirá que "No ay arte o sciencia en letras apartada, / qu'el vulgo no la tenga decorada»- ${ }^{13}$ Esta antigua tradición escolar y este contexto humanista de jerarquización de la lengua castellana y revalorización de las expresiones populares es también el ambiente que vive este comentarista a mediados del siglo XVI y esto explica su preferencia en seleccionar los refranes de Celestina. Partamos de la base que nos faltan declaraciones personales del autor de los propósitos que perseguía con este comentario. Tal vez ellas estuvieran en los folios iniciales del manuscrito, actualmente perdidos. Por tanto, sólo tenemos su labor como para deducir cuáles hayan sido sus intenciones.

Veamos una vez más su método de glosa. En el tercer acto el comentarista glosa el refrán "Todo lo puede el dinero» que en la Tragicomedia desencadena una larga reflexión de la alcahueta. Se trata de un difundido refrán que el comentador enlaza con el Eclesiástico (en verdad el Eclesiastés 10: 19): «Pecuniae omnia obediunt» y con un pasaje de Horacio: "Omnis enim res, virtus, fama, decus divina humanaque pulchris divitis parent, quas qui construxerit, ille clarus erit, fortis, justus, sapiens etiam et rex, et quicquid volet» (pp. 165-166). No es que el comentarista ignore versiones populares de este refrán, ${ }^{14}$ pero su interés es el de asimilarlo a dichos bíblicos y de la Antigüedad. Concuerdo con la afirmación de Russell en que el comentarista quería llamar la atención de que las opiniones, sen-

11.- Remito para ello al capítulo 5 de mi libro El refranero castellano en la Edad Media, Madrid, Ediciones Laberinto, 2004, pp. 105-110.

12.- Ellas iban a aflorar en opúsculos como la Epistola exhortatoria a las letras de Juan de Lucena, el Libro de las alabanças de las lenguas hebrea, griega, latina, castellana y valenciana de Martín de Viciana (Valencia, 1574) o el capítulo final de la obra de Bernardo Aldrete, Del origen y principio de la lengua castellana o romance qui oi se usa en España (Roma, 1606), así como en multitud de prólogos del período. Véase al respecto el capítulo de Ángel Gómez Moreno, «El pulso de la lengua vulgar con las clásicas», en España y la Italia de los Humanistas, Madrid, Gredos, 1994, pp. 109-120.

13.- Juan de Mal Lara, La philosophía vulgar. Ed. Manuel Bernal Rodríguez, Madrid, Biblioteca Castro, 1996, p. 7.

14.- Que se documentan en Castilla desde el primer tercio del siglo XIV. Vid. Eleanor S. O'Kane, Refranes y frases proverbiales españolas de la Edad Media, Madrid, RAE Anejo II, 1959, p. 103 y Hugo O. Bizzarri, «Dádivas quebrantan peñas» en Carlos Alvar (coord.), Gran enciclopedia cervantina, t. Iv, Madrid, Castalia-Centro de Estudios Cervantinos, en prensa. 
tencias y exempla de la Tragicomedia reflejaban el saber colectivo de las autoridades reconocidas. ${ }^{15}$ Pero es necesario puntualizar que no es ésta una actitud personal del comentador, sino una de las técnicas que los humanistas tenían para dignificar los textos vulgares y los dichos populares. En los studia humanitatis los florilegios de refranes y sentencias tenían una clara función didáctica apoyada en el criterio de la autoridad. Este refrán en sí no necesita de mucha explicación, pues, entre otras cosas, tocaba un tópico de la Edad Media, pero le sirve al comentarista para hacer una libre reflexión sobre el poder del dinero, reflexión que salta de autoridad en autoridad: Horacio, Petronio, Menandro, Ovidio, Terencio, Aristóteles, Boecio, Giovanni Nevizzano. Todo estos autores no hacen más que refrendar lo que aquí ha querido decir Rojas con este refrán: "Ansi que mui bien e con justa razon pudo dezir aqui nuestro author que el dinero lo puede todo» (p. 167), finaliza diciendo

De una forma u otra el comentarista siempre quiere relacionar lo dicho en el texto con fuentes clásicas. Del refrán "Muertas si cansadas no", que Celestina coloca para caracterizar la insaciabilidad amorosa de las mujeres burguesas, comenta: «Estas son quasi las palabras que dize Juvenal Satyra 6 hablando de Messalina mujer del emperador Claudio: Et lassata que viris nondum saciata recessit (Cansada aunque no del todo harta de los hombres se aparto)» (p. 169). Remite luego a la glosa de Juan de Mena hecha por Hernán Núñez y a la Summa de Antonius Florentinus, para agregar de su propia cosecha: «Y esto es porque de su naturaleza las mujeres en la concuspisencia de la carne por la maior parte son insaciables» (p. 169), lo que luego apoyará con dichos de Salomón. Cuando en el mismo acto Rojas menciona a las Tres furias (Tesífone, Megera y Aleto) el comentarista señala: «Para entendimiento de estas palabras emos de saber que como quenta la Margarita de los poetas..." (p. 173) para explicar la referencia mitológica. U otras veces su glosa comienza: «En estas palabras nos quiere nuestro author dar a entender...» (p. 205). A cada paso se evidencia que el comentarista quiere desentrañar el sentido del texto y sacar sus valores morales.

Pero como frecuentemente ocurre en la exégesis gramatical, no siempre un texto tiene un único sentido. En estos casos el comentarista reconoce un sentido literal y otro profundo:

Que te hizo alcalde mengua de hombres etc. Este refran se entiende en dos maneras y la una de ellas quiere dezir como comunmente se toma que por falta de hombres buenos que no avia otro mejor que pudiesse ser alcalde o juez que tu por tanto lo eres y te hizieron juez que de otra manera no lo fueras y en este sentido aquí esta puesto e se a de entender queriendo dezir que no mere- 
cia ser juez sino porque no avia otro mejor que lo fuesse [...] Pero el otro y segundo sentido que tiene este refran es mejor a mi juizio y mas verdadero aunque en el proposito que aquí tratamos no convenga que quiera dezir quien te hizo alcalde falta de hombres buenos porque si hombres buenos no faltaran y todos fueron buenos y en ellos no oviera falta, no avia necessidad de alcaldes ni justicia y en este sentido lo dize el Philosopho como lo refiere la 1 . I $\mathrm{tt}^{\circ} 27$ Partida 4 que dize ansi estas palabras (p. 409).

Pero en la mayoría de los casos el comentarista hace una explicación literal $y$, en algunos, hasta ingenua, como la del refrán "Un dolor saca otro»: "Esta es proposición de medicina que tienen los medicos ser imposible que dos dolores esten juntamente en un mesmo lugar sino que el uno saca al otro como ya diximos arriba en el acto 10 en la glosa 13» (p. 500).

Otro aspecto hasta ahora desatendido de este comentario son los vastos conocimientos lingüísticos de que hace gala el glosador. Identifica que muchos refranes que coloca Rojas provienen de la tradición latina. Así "La presta dádiva su effecto a doblado» proviene de Terencio "Qui sero dat nihil dat et qui cito bis dat» (p. 124), "Mal me quieren mis comadres...» también de Terencio "Osequium amicos, veritas odium parit» ( $p$. 155), o "A esse tal dos alevosos. En latin se dize este refran: Fallatia alia aliam trudit» (p. 165), "Que no se toman trucha etc. Vulgarmente se dize en latin: Nihil sine labore fit» (p. 273), «Ni es todo oro quanto amarillo etc. La Margarita de los poetas en la foja 48 en el fin dize que es viejo proverbio o sentencia esto que aquí se dize: Non omne quod licet aurum judicari debet» (p. 318), "Un clavo con otro se expelle. Dizese en latin comunmente esto: Clavus clavo expellitur» (p. 355), "Aquel va mas sano que va por llano. Esto es proverbio comun que en latin se dize: Qui vadit plane, vadit sane» (p. 368). O simplemente ve que un refrán castellano concuerda con uno latino, como es el caso de "El hombre apercibido. Con este refran concuerda y haze en semejante lo que en latin se dize comunmente: Tela nocent levius visa volare prius» (p. 371), "Que criasse cuervo etc. Con esto conforman las palabras que dize el texto en el cap. et si Judeos de Judeis onde dize que es comun proverbio: Mus in pera, serpens in gremio, et ignis in sinu suis consueverunt hospitibus exhibere (El mur en el çurron y la serpiente en el regaço y el fuego en el seno acostumbraron de mal galardonar a sus huespedes)» (p. 411), "A otro perro con esse huesso. Dizese en latin este refran : Ad populum phaleras ego te intus et in cute novi» (p. 463). Esta labor de filólogo y anotador que lleva a cabo el comentarista de Celestina es para dar a entender que los refranes expresados en vulgar en esta obra provienen, en realidad, del latín. Como los gramá- 
ticos sostenían en la época el vulgar era tan sólo un latín corrompido ${ }^{16}$. Esta manera de identificar refranes vulgares y latinos tampoco es un rasgo personal de este comentarista, sino una técnica de los studia humanitatis. Juan de Palmireno en su De vera et facile imitatione Ciceronis (Teruel, 1560) coloca una lista de 197 refranes con los que quiere enseñar a su discípulo que no se puede traducir un refrán literalmente y para ello elige paralelos la mayor parte tomados de Erasmo. ${ }^{17}$

En las diversas glosas el comentarista revela la diferente percepción lingüística que tenía de la fraseología de Celestina. Algunas formas no duda en calificarlas de refranes: "No ai cosa tan fuerte que con dinero no se puede combatir. Y esto que aqui etc. Este dicho lo llaman refran viejo» (p. 168), "Lo incognito por lo menos conoscido. Esta es una manera de hablar o como refran latino que se dize: Ignotum per ignotius» (p. 184). En un caso indica sin decirlo directamente que se trata de un refrán: «Y ansi dezimos comunmente el amor sin temor no es amor» (p. 398). En ocasiones la voz refrán no indica un dicho popular sino popularizado: "Con mal anda el uso quando la barva etc. Este refran o sentencia aunque comunmente se dize algunos no lo entienden propiamente lo que por el se quiere dezir» (p. 209). A mediados del siglo xvI, cuando era común que circularan en forma manuscrita e impresa colecciones de refranes, para el comentador de Celestina el refrán tiene una forma expositiva determinada. Por eso de "Que es alivio a los miseros etc.», dice: «Este es un dicho a manera de refran que entre los latinos se quenta e dize de esta manera: Solatium est miseris socios habere pennarum» (p. 505). "Nunca haras casa con sobrado" parece considerarla en otra categoría, en nuestra nomenclatura una locución: «Quiere dezir que nunca llegan riquezas que con ellas pudiesse hazer buenas casas. Es manera de dezir» (p. 293). «Seria quitar de un sancto por poner otro» (p. 301) lo califica como «dicho que vulgamente lo solemos ansi dezir aun en derecho" y como "manera de hablar» (p. 301). Gracias al comentarista nos enteramos que el sintagma "La pena le hara querdo» formaba parte de la fraseología de la época: «Esto es dicho comun y vulgar» (p. 390).

Esta percepción lingüística que ejercita el comentarista hace entrar otro elemento en sus comentarios: la experiencia personal de la que se vale para interpretar, por ejemplo, la frase proverbial "Calças de Villadiego»: «Porque se diga tomo calças de Villadiego quando queremos dezir por buenos terminos que uno echo a huir, creo es porque aquel pueblo Villadiego esta asentado en un mui gran llano por donde se huie e corre mui bien e ansi por esto se dice tomo calças de Villadiego por dezir echo mui

16.- Así lo expresa claramente Sebastián de Covarrubias en el prólogo al lector de su Tesoro de la lengua castellana. Vid. Avelina Carrera de la Red, El problema de la lengua en el Humanismo Renacentista español, Valladolid, Universidad de Valladolid, 1988, p. 73.

17.- Germán Colón Domènech, «Los Adagia de Erasmo en Español (Lorenzo Palmireno, 1560) y en Portugués (Jerónimo Cardoso, 1570)», RFE, 84 n 1 (2004), pp. 5-27. 
bien a huir ligeramente sin aver que le estorvasse» (p. 373). Al comentar "La obra sobrepuja a la materia», además de asimilarlo a un refrán latino, utiliza sus observaciones lingüísticas: "Y en la puente de Alcantara estan unos versos que dizen lo mismo para encarecer mas el artificio y hechura que el... de ellas» (p. 123). También da una perfecta explicación de "No me avian dexado gota de sangre» (p. 379) y de «Cargado de hierro cargado de miedo» (p. 380).

De entre sus comentarios lingüísticos no hay que dejar de lado algunos de carácter etimológico, como el que dedica a «Una legua» (pp. 425-427), medida que el comentarista define en toda su variedad románica, aunque aclara que «[...] la legua castellana propiamente tiene en si tres millas como se prueba bien e lo quiso ansi dezir aunque por otras palabras la 1. 3 $\mathrm{tt}^{\circ} 16$ Partida 2» (p. 425). La glosa no deja de definir otras medidas como mansum y dieta haciendo una clara distinción entre lengua latina y castellana: "[...] digo que dieta o jornada en romance segun derecho se a de entender e contiene por tierra diez leguas de las nuestras castellanas» (p. 426). Lo mismo puede decirse de su interpretación del nombre de Celestina (p. 58), de la voz "combite» (p. 324) o del giro «estar la cabeçera en la mesa» (p. 325).

El último recurso lingüístico que querría señalar es la adición de nuevos refranes en las glosas. Dieciséis son los refranes que agrega. ${ }^{18}$ La técnica no es nueva. Ya en el Seniloquium se puede encontrar este fenómeno en el que los refranes se codean con citas de los más prestigiosos autores de la Antigüedad. El procedimiento se podría rastrear en glosas a textos ya del siglo XI en Francia. ${ }^{19}$ Pero volviendo a nuestro comentario, el comentarista explica el mote de «barbuda» dado a Celestina con un refrán:
Llamala barbuda el author para denotar e manifestar quan mala era que aun aca comunmente dezimos: A la mujer barbuda de aparte la saluda dando consejo que no nos lleguemos a su tracto. Y en latin tanbien se dize: Mulier de longe salutata» (p. 58).

18.- Doy su listado: "A la mujer barbuda de aparte la saluda» (p. 58), "Quan lexos de ojos quanto de coraçon» (p. 60), "Ojos que no veen coraçon que no quiebran» (p. 60), «Al buen entendedor pocas palabras» (p. 66), «El pece por huir de la sarten cae en las brasas» (p. 67), «Mas vale un buen amigo que el dinero del arca» (p. 94), "Mas veen dos ojos que uno» (p. 122), "Por la experiencia se hagan los hombres arteros y sabios» (p. 288), «El perro del ortelano que ni el come las verças y a envidia y enojo que otr las coma" (p. 288), "Obras son amores y no buenas razones» (p. 292), "Hombre aperçebido medio combatido» (p. 318), «Cosa de gran trabajo es suffrir la prosperidad» (p. 365), «Mas antes es otra mesma la naturaleza» (p. 433), "otra cualquier mudança [...] es a par de muerte» (p. 433), "Quando la barba de tu vezino vieres pelar echa la tuia en remojo» (p. 439), "[...] si el molinero es sordo».

19.- Vid. al respecto Elisabeth Schulze-Busacker, «La constitution des recueils de proverbes et de sentences dans l'Antiquité tardive et le Moyen Àge», en La transmission de savoirs au Moyen Àge et à la Rennaissance. t. I. Du XII au XV siècle, sous la direction de Pierre Nobel, Besançon, Presses Universitaires de Franche-Comté, 2005, pp. 259-287. 
En un caso indica que una frase de Alisa alude a un refrán: «Nuestras barba en remojo. Es lo que comunmente dezimos: Quando la barba de tu vezino vieres pelar echa la tuia en remojo» (p. 439). En otras completa un refrán que solía difundirse en dos versiones, breve y extensa: «Por demas es la citola en el molino etc. Quiere dezir i mas si el molinero es sordo como comunmente se dize» (p. 445); «No seas el perro del hortelano [...] $Y$ ansi dezimos el perro del ortelano que ni el come las verças y a envidia y enojo que otro las coma» (p. 288). Para comentar la sentencia de Sempronio (acto I) con la cual el sirviente justifica sus tres días de ausencia de la casa de Elisa, el comentarista coloca dos refranes de gran difusión en ese momento aún entre los poetas líricos que hablan de la pena de amor: "Y comunmente dezimos que lexos de ojos quanto de coraçon, y ojos que no ven coraçon no quiebran» (p. 60) para comentar cómo la ausencia destruye las amistades. El comentarista se ha marcado el objetivo de explicar el sentido profundo del texto de Rojas. Es por eso que en ocasiones recurre a refranes para explicar sentencias que, diciendo lo mismo con palabras más sencillas, están más cercanos a la comprensión de sus futuros lectores: «Cae en otro maior. Este propiamente es refran latino y ansi se dize: Incidit in Cyllam cupiens vitare Carybdem [...] y para mas declaracion en romance tanbien dezimos el pece por huir de la sarten cae en las brasas» (p. 67). Puede darse el caso de que con un refrán comente otro refrán: "No lo se a las obras creo. Quiere en effecto dezir en estas palabras lo que por otras comunmente dezimos que obras son amores y no buenas razones» (p. 292). Otras veces los refranes pueden ayudar a sus comentarios lingüísticos: «Que basta para mi mecer el ojo. Es esto lo que dezimos al buen entendedor pocas palabras» (p. 66).

Una última consideración sobre este copioso comentario. Los criticos han señalado con justeza que el comentarista no hace apreciaciones sobre el valor estético de La Celestina. Más bien, sólo parecen interesarle de ella sus valores morales. Y en especial Russell ha destacado la costumbre del comentador de citar texto latino y ofrecer a continuación su traducción. «De dicha costumbre quizá pueda inferirse que, por la razón que fuera, quería que su comentario fuera accesible a los que no podían leer latín con facilidad». ${ }^{20}$ No es descartable esta hipótesis, pero también hay que tener en cuenta que la presentación de un texto en su lengua original y su inmediata traducción era técnica frecuente entre los humanistas que de esta forma colocaban ambas lenguas al mismo nivel. ${ }^{21} \mathrm{El}$ comentarista no

\section{0.- Russell, «El primer comentario...», p. 299.}

21.- Sobre la disputa latín-vulgar, vid. A. Morel-Fatio, «L'espagnol langue universelle», BHi, 15 (1913), pp. 207-225 ; E. Buceta, "La tendencia a identificar el español con el latín», en Homenaje ofrecido a Menéndez Pidal, t. I, Madrid, Hernando, 1925, pp. 85-108; M. Romera Navarro, «La defensa de la lengua española en el siglo XVI», BHi, 31 (1929), pp. 204-255; O. Green, "Literatura española: Desde la inferioridad hasta la igualdad", en España y la tradición occidental. El espíritu castellano en la literatura desde El Cid hasta Calderón, t. III, Madrid, Gredos, 1969, pp. 
hace apreciaciones estéticas de la Tragicomedia, (los humanistas no solían hacerlas, más bien en sus comentarios se interesaban por desentrañar el sentido literal del texto o de explicar palabras), ${ }^{22}$ pero no por eso desdeña la lengua en la cual está escrita. Todo su comentario se preña de ese sentimiento de dignificación de la lengua vulgar que poseían los humanistas. De no haber sido así no le habrían interesado los refranes ni los giros que comenta. Las más de cuarenta oportunidades en las que cita a Hernán Núñez son en este caso significativas y tal vez podrían darnos la pista de su formación humanística. ${ }^{23}$ Como el Comendador griego hizo con Juan de Mena, tal vez su intención de comentar la obra de Rojas fuera la de darle el reconocimiento de obra "clásica».

He dejado intencionalmente de hacer referencia a los conocimientos de Derecho de nuestro comentarista por haber sido suficientemente puestos en evidencia por Russell y Fernández Rivera. Al costado de ese rasgo profesional que aflora en las glosas del anónimo comentarista de Celestina, es necesario señalar que su comentario se destaca por un interés en el texto de Rojas. Y en el caso de explicar los refranes es necesario advertir que estas glosas no se diferencian en su forma de interpretar las paremias al método empleado por el anónimo comentarista del Seniloquium o de Mal Lara. Su inserción en este caso en los studia humanitatis es innegable, estudios que, como bien se ha indicado, no eran ajenos a la formación de un jurista. ${ }^{24}$

287-318; O. Di Camillo, «Nebrija y la afirmación del humanismo español», en El humanismo castellano del siglo XV, Valencia, Fernando Torres Editor, 1976, pp. 269-296; D. Ynduráin, «La invención de una lengua clásica (literatura vulgar y renacimiento en España)", Edad de Oro, 1 (1982), pp. 13-34; P. Ruiz Pérez, "Sobre el debate de la lengua vulgar en el renacimiento», Criticón, 38 (1987), pp. 15-44; A. Carrera de la Red, El problema de la lengua en el Humanismo renacentista español, Valladolid, Universidad de Valladolid, 1988.

22.- El único juicio de valor que se le escapa es referido a las Coplas de Jorge Manrique: "Que aunque en romance no ai obra tan subida e de tan galanas sentencias en su materia e proposito» (p. 505). Sobre la tarea interpretativa de los Humanistas, vid. W. Keith Percival, "Renaissance Grammar», en Albert Rabil Jr. (ed.), Renaissance Humanism. Foundations, Forms, and Legacy. t. III. Humanism and the Disciplines, Philadelphia, Pennsylvania UP, 1988, pp. 67-83.

23.- Vid. su estudio en Michel García, «Las fuentes literarias castellanas del glosador de Celestina», Celestinesca, 21 n 1-2 (1997), pp. 49-64.

24.- En palabras de W. Keith Percival («Renaissance Grammar», p. 68): «The liberal arts, however, did not stand on their own but were regarded as a preparation for more advanced study, namely, for one of the two learned professions, law and medicine». 


\section{Bibliografía}

Alvar, Carlos (coord.), Gran enciclopedia cervantina, Madrid, Castalia-Centro de Estudios Cervantinos, 2005.

BizzArRi, Hugo O., El refranero castellano en la Edad Media, Madrid, Ediciones Laberinto, 2004.

BUCETA, Erasmo, "La tendencia a identificar el español con el latín», en Homenaje ofrecido a Menéndez Pidal, t. I, Madrid, Hernando, 1925, pp. 85-108.

Cantalapiedra Erostarbe, Fernando, "La Celestina y Seniloquium», Celestinesca, 29 (2005), pp. 9-44.

Cantalapiedra, Fernando y Juan Moreno (eds.), Diego García de Castro. Seniloquium, Valencia, Universidad de Valencia, 2006.

Carrera de la Red, Avelina, El problema de la lengua en el Humanismo Renacentista español, Valladolid, Universidad de Valladolid, 1988.

Colón DomèneCH, Germán, "Los Adagia de Erasmo en Español (Lorenzo Palmireno, 1560) y en Portugués (Jerónimo Cardoso, 1570)», RFE, 84 $\mathrm{n}^{\circ} 1$ (2004), pp. 5-27.

Corfis, Ivy, "La Celestina comentada y el código jurídico de Fernando de Rojas", en A. Deyermond y I. Macpherson (eds.), The Age of the Catholic Monarchs 1474-1516: Literary Studies in Memory of Keith Whinnom. Número especial del Bulletin of Hispanic Studies, Liverpool, Liverpool UP, 1989, pp. 19-24.

Di Camillo, Ottavio, El humanismo castellano del siglo xv, Valencia, Fernando Torres Editor, 1976.

Faulhaber, Charles B., "Celestina de Palacio: Madrid, Biblioteca de Palacio, Ms. 1520", Celestinesca, 14 n 2 (1990), pp. 3-39.

-, "Celestina de Palacio: Roja's Holograph Manuscript?» Celestinesca, $15 \mathrm{n}^{\circ}$ 1 (1991), pp. 3-52.

Fernández Rivera, E., "La autoría y el género de la Celestina comentada» $R F E, 86 \mathrm{n}^{\circ} 2$ (2006), pp. 259-276.

Fothergill-PaYne, Louise, Enrique, Fernández Rivera y Peter FothergillPayne (eds.), Celestina comentada, Salamanca, Ediciones Universidad de Salamanca, 2002.

GarCiA, Michel, "Las fuentes literarias castellanas del glosador de Celestina», Celestinesca, 21 no 1-2 (1997), pp. 49-64.

Gómez Moreno, Ángel, España y la Italia de los Humanistas, Madrid, Gredos, 1994.

Green, Otis, España y la tradición occidental. El espíritu castellano en la literatura desde El Cid hasta Calderón, t. III, Madrid, Gredos, 1969.

Mal Lara, Juan de, La philosophía vulgar. Ed. Manuel Bernal Rodríguez, Madrid, Biblioteca Castro, 1996. 
Morel-Fatio, Alfred, «L'espagnol langue universelle», BHi, 15 (1913), pp. 207-225.

O'KANE, Eleanor S., Refranes y frases proverbiales españolas de la Edad Media, Madrid, RAE Anejo II, 1959.

Percival, W. Keith , «Renaissance Grammar», en Albert Rabil Jr. (ed.), Renaissance Humanism. Foundations, Forms, and Legacy. t. III. Humanism and the Disciplines, Philadelphia, Pennsylvania UP, 1988, pp. 67-83.

ROMera NAVARRO, M., "La defensa de la lengua española en el siglo XVI», BHi, 31 (1929), pp. 204-255.

Ruiz PÉREZ, P., "Sobre el debate de la lengua vulgar en el renacimiento», Criticón, 38 (1987), pp. 15-44.

RusselL, Peter, «El primer comentario crítico de La Celestina: cómo un legista del siglo XVI interpretaba la Tragicomedia», en Temas de La Celestina y otros estudios. Del Cid al Quijote, Barcelona, Ariel, 1978, pp. 293-321.

—, "La Celestina y los estudios jurídicos de Fernando de Rojas", en Temas de La Celestina y otros estudios. Del Cid al Quijote, Barcelona, Ariel, 1978, pp. 325-340.

SCHUlZE-BusACKeR, Elisabeth, "La constitution des recueils de proverbes et de sentences dans l'Antiquité tardive et le Moyen Àge», en La transmission de savoirs au Moyen Àge et à la Rennaissance. t. I. Du XII au $X V^{e}$ siècle, sous la direction de Pierre Nobel, Besançon, Presses Universitaires de Franche-Comté, 2005, pp. 259-287.

YNDURÁin, Domingo, "La invención de una lengua clásica (literatura vulgar y renacimiento en España)», Edad de Oro, 1 (1982), pp. 13-34.

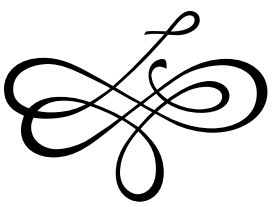




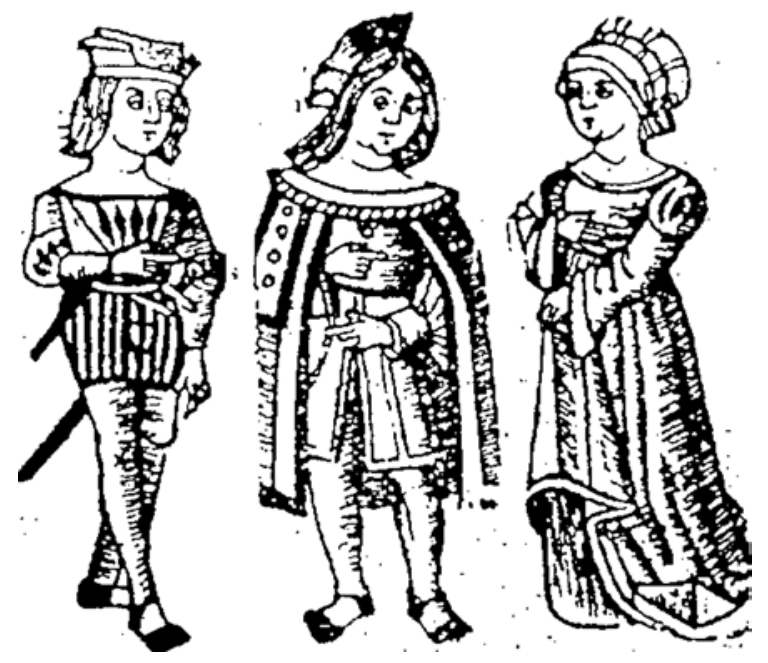




\section{BizARRI, Hugo O., "Los refranes de Celestina interpretados por su primer comentarista», Celestinesca 31 (2007), pp. 9-22.}

\section{RESUMEN}

El compendio conocido como Celestina comentada ha sido analizado en repetidas oportunidades para conocer esta particular forma de recepción de la obra. Específicamente, se ha destacado su relación con el ámbito del derecho al conjeturarse que ha sido escrita por un abogado imbuido por la corriente del mos gallicus que estaba de moda a mediados del siglo XVI. En el presente trabajo se pretende profundizar otro aspecto: la concepción que este anónimo comentarista tenía de la lengua castellana. En este sentido, se acentúa la relación del autor con los studia humanitatis de donde proceden muchos de sus rasgos de comentario: la explicación de giros, la recurrencia a etimologías, la equiparación de la lengua vulgar a la latina, su consideración de la obra como clásico, entre otros. En definitiva, se postula que este comentario no se diferencia en su forma de interpretar las paremias a la que realizaron obras como el anónimo Seniloquium o la Philosophía vulgar de Juan de Mal Lara. Su inclusión, por tanto, en los studia humanitatis es indudable.

PALABRAS ClAVE: Celestina comentada, Seniloquium, refranes.

\section{ABSTRACT}

The compendium known as Celestina comentada has been analyzed on several occasions with the notion of understanding this rather special form of the reception of Celestina. Specifically of interest has been its nexus with a legal mindset, for it has been proposed that it was carried out by a lawyer versed in the mos gallicus so much in fashion in the mind sixteenth century. In this study, I examine a different aspect of the work: the author's conception of the Spanish language. Thus, I emphasize the author's relationships to the studia humanitatis from with so many of the characteristics of his comentary derive: the explanations of turns of phrase, the recourse to etymologies, the comparisons of the common language to te latin language and the treatment of the work as alrady a classic, among other characteristics. I propose that the commentary differs little from the interpretation of proverbial expressions realized in such works as the anonymous Seniloquium or the Philosophia vulgar of Juan de Mal Lara. Its ascription, then, to the studia humanitatis, is not to be doubted.

KEY WORDS: Celestina comentada, Seniloquium, refranes. 